\title{
Efficacy of ranibizumab combined with photodynamic therapy on wet age-related macular degeneration
}

\author{
LING CHEN $^{1 *}$, BING WANG $^{2^{*}}$, WEI CUI ${ }^{3}$ and SHUFEN FANG ${ }^{4}$ \\ ${ }^{1}$ Department of Ophthalmology, Affiliated to Qingdao University Yuhuangding Hospital of Yantai; \\ ${ }^{2}$ Department of Ophthalmology, Yantaishan Hospital, Yantai, Shandong 264000; ${ }^{3}$ Department of \\ Ophthalmology and Otolaryngology, Qingdao Fuwai Cardiovascular Disease Hospital, Qingdao, \\ Shandong 266034; ${ }^{4}$ Department of Ophthalmology, Laizhou People's Hospital of Yantai, \\ Yantai, Shandong 261400, P.R. China
}

Received October 23, 2019; Accepted December 19, 2019

DOI: $10.3892 /$ etm.2020.8641

\begin{abstract}
Efficacy and safety of intravitreal ranibizumab (IVR) combined with photodynamic therapy (PDT) in treating wet age-related macular degeneration (wAMD) were studied. A total of 130 eyes were collected from 130 wAMD patients treated in Affiliated to Qingdao University Yuhuangding Hospital of Yantai, of which 65 were given IVR combined with PDT (combination therapy group) and the remaining 65 were treated with simple IVR (ranibizumab group). The differences in best corrected visual acuity (BCVA), central macular thickness (CMT), intraocular pressure, choroidal neovascularization $(\mathrm{CNV})$ leakage, levels of serum vascular endothelial growth factor (VEGF) and transforming growth factor- $\beta 1$ (TGF- $\beta 1$ ) as well as complication rate were compared before and after treatment between the two groups. At 1, 3, 6 and 12 months after treatment, combination therapy group had remarkably better BCVA and notably smaller CMT than ranibizumab group. Fundus fluorescein angiography (FFA) showed that the area of macular degeneration was reduced markedly after treatment in both groups, and the area in combination therapy group was evidently smaller than that in ranibizumab group at 1,3 and 6 months after treatment. At 3 months after treatment, the levels of serum VEGF and TGF- $\beta 1$ declined obviously in the two groups compared with those before treatment. The IVR combined with PDT can effectively improve the visual acuity, decrease CMT and prominently reduce the area of macular degeneration of
\end{abstract}

Correspondence to: Dr Shufen Fang, Department of Ophthalmology, Laizhou People's Hospital of Yantai, 1718 Wuli Street, Laizhou, Yantai, Shandong 261400, P.R. China

E-mail: shufan122@163.com

${ }^{*}$ Contributed equally

Key words: age-related macular degeneration, photodynamic therapy, ranibizumab
wAMD patients, and its therapeutic effects are long-standing and tolerable for the patients, so it is worthy of clinical popularization.

\section{Introduction}

Wet age-related macular degeneration (wAMD) is a common cause of blindness in clinic. Molecular biological studies have discovered that the imbalance between pro-angiogenic factors and anti-angiogenic factors is the leading cause of wAMD, in which vascular endothelial growth factor (VEGF) is highly expressed, and choroidal neovascularization (CNV) is formed, resulting in visual loss of the patients $(1,2)$. Ranibizumab, a kind of monoclonal antibody fragment, belongs to angiogenesis inhibitors and achieves its therapeutic purposes by preventing vascular leakage and blocking choroidal vessels (3).

Photodynamic therapy (PDT) is a new therapy for CNV in recent years (4), which can protect the peripheral normal tissues to the greatest extent while blocking CNV. During PDT, the lesion site is irradiated by laser of a specific wavelength, and the activated photosensitizing agent produces photooxidation reaction to kill endothelial cells and selectively destroy aberrant neovascularization, without damaging surrounding normal retina (5). Studies have demonstrated that ranibizumab combined with PDT has preferable efficacy in treating wAMD (6-8).

In this study, the clinical data of 130 eyes of 130 patients with wAMD treated in Affiliated to Qingdao University Yuhuangding Hospital of Yantai (Yantai, China) from May 2017 to May 2018 were retrospectively reviewed, and the efficacy and safety of simple intravitreal ranibizumab (IVR) and IVR combined with PDT in treating wAMD were comparative analyzed, with the aim to provide a more scientific basis for formulating efficacious treatment protocols.

\section{Patients and methods}

General data. A total of 130 eyes of 130 wAMD patients who were treated in the hospital from May 2017 to May 2018 were 
selected as the objects, which were divided into combination therapy group (IVR combined with PDT, $\mathrm{n}=65$ ) and ranibizumab group (simple intravitreal injection of ranibizumab, $\mathrm{n}=65$ ) using a random number table. Inclusion criteria: patients aged $\geq 50$ years, those with monocular lesion and those clinically diagnosed with exudative AMD. Diagnostic criteria: i) visual deterioration (or accompanied with metamorphopsia), macular drusen, depigmentation or repigmentation and typical CNV signs, ii) subretinal hemorrhage, exudate and fibrotic membrane, iii) best corrected visual acuity (BCVA) $\geq 25$ letters before treatment, and iv) basically clear refractive media, without influences on fundus examination and fundus fluorescein angiography (FFA). Exclusion criteria: i) patients whose CNV in the trial eye was previously treated via intravitreal injection of drugs, PDT, transpupillary thermotherapy (TTT), retinal laser photocoagulation, macular grid photocoagulation or submacular surgery; ii) those with ocular tumor, trauma, glaucoma, or eye infection, iii) those with high myopia, iv) those complicated with other types of retinal diseases (polypoidal choroidal vasculopathy or dry AMD), v) those with severe cardiac, pulmonary hepatic or renal dysfunction, or vi) those who had serious hypersensitivity reactions to the medicines applied in this study. There were 70 males and 60 females aged 51-82 years, with a course of disease of 3-21 months. The differences in the age, sex, course of disease, baseline visual acuity, central macular thickness (CMT) and CNV type and location were not statistically significant between the two groups (Table I). This study was reviewed and approved by the Ethics Committee of Affiliated to Qingdao University Yuhuangding Hospital of Yantai, and the patients and their families were informed and signed the informed consent.

Therapeuticmethods.IVR: The eyes were instilled with of loxacin eye drops (Tarivid, Santen Pharmaceutical Co., Ltd.) 3 days before operation (4 times/day). The operation was performed under sterile conditions. The patients were in the supine position, underwent routine disinfection and draping and received oxybuprocaine hydrochloride eye drops (Benoxil, Pharmaceutical Co., Ltd.) for topical anesthesia of the eyeball, followed by flushing of the conjunctival sac with $5 \%$ povidone iodine and normal saline. Then a $30 \mathrm{G}$ needle was vertically inserted into the vitreous cavity through the pars plana of ciliary at $3.5 \mathrm{~mm}$ away from the corneoscleral limbus toward 10 o'clock, and $0.5 \mathrm{mg}(0.05 \mathrm{ml})$ of ranibizumab (Lucentis, Novartis AG) was injected slowly. After operation, the eye was packed with Tarivid eye ointment. The intraocular pressure was closely monitored at $2 \mathrm{~h}$ after operation, and the eye was given Tarivid eye drops 4 times/day for 1 consecutive week to prevent postoperative infection. Course of treatment: once a month for 3 consecutive months.

IVR combined with PDT: The photosensitizer verteporfin (Visudyne, Novartis AG) $\left(6 \mathrm{mg} / \mathrm{m}^{2}\right)$ was diluted to $30 \mathrm{ml}$ using 5\% glucose and intravenously injected in a dark room within $10 \mathrm{~min}$. Fifteen minutes later, the macular degeneration was persistently irradiated by laser at the wavelength of $689 \mathrm{~nm}$ for $83 \mathrm{sec}$, with an intensity of $600 \mathrm{~mW} / \mathrm{cm}^{2}$ and a density of $50 \mathrm{~J} / \mathrm{cm}^{2}$. The eye was protected against light within 5 days after treatment. At 3 days after PDT, ranibizumab was intravitreally injected once according to the same mode of injection and course of treatment.

Observation indexes. The FFA, BCVA examination, optical coherence tomography (OCT) and color fundus photography were performed for the two groups of patients before treatment and at 1,3 and 6 months after treatment. As for BCVA examination, the standard logarithmic visual acuity chart was employed, and the results were converted to logarithm of the minimal angle resolution ( $\log$ MAR) visual acuity during statistics. Kowa nonmyd $\alpha$-DIII non-mydriatic fundus camera was used for color fundus photography, and Spectralis HRA was utilized for FFA. Spectralis OCT instrument was adopted for OCT, and horizontal linear scanning was performed centered on the central fovea of macula (scanning depth, $1.9 \mathrm{~mm}$; scanning area, $6 \mathrm{~mm} \times 6 \mathrm{~mm}$; lateral resolution, $14 \mu \mathrm{m}$; axial resolution, $7 \mu \mathrm{m}$; and scanning mode, $512 \times 496)$. The distance from the inner limiting membrane of retinal neurosensory layer to the lateral high reflection band of the retinal pigment epithelium layer was measured using the built-in locating software of the instrument, which was regarded as the CMT. The measurement was completed by two experienced technicians and repeated 3 times to calculate the average value. The FFA combined with ICGA was carried out at 3 and 6 months after treatment to determine the area of macular degeneration, so as to reflect the CNV leakage. The degree of CNV leakage was classified as progressive (the lesion area is increased compared with that before treatment), stable (the lesion area is slightly increased or decreased compared with that before treatment), improved (the lesion area is decreased by $<1 / 2$ ) and ameliorated (the lesion area is decreased by $>1 / 2$ ), of which stable, improved and ameliorated grades are all considered as effective treatment.

Criteria of IVR retreatment: The retinal thickness in the macular region is increased by $>100 \mu \mathrm{m}$, with subretinal fluid and retinal edema, and active CNV is detected by FFA when there is new retinal hemorrhage or short-term diminution of visual acuity by 5 letters.

The BCVA, change in mean CMT, change in intraocular pressure, CNV leakage and occurrence of complications were recorded before treatment and at 1,3 and 6 months after treatment. Moreover, $5 \mathrm{ml}$ of fasting venous blood was drawn from every patient in the two groups before treatment and at 3 months after treatment separately to detect and compare the levels of VEGF and transforming growth factor- $\beta 1$ (TGF- $\beta 1$ ) via enzyme-linked immunosorbent assay.

Statistical analysis. Statistical Product and Service Solutions (SPSS) 22.0 (IBM) was adopted for statistical analysis. The enumeration data were presented as ratio (\%), and $\chi^{2}$ test was conducted for inter-group comparison. The measurement data were expressed as mean \pm standard deviation (mean $\pm \mathrm{SD}$ ), and repeated measures analysis of variance was applied to compare the BCVA, CMT and intraocular pressure of the patients at different time points, independent-samples t-test was performed for comparison between groups, and least significant difference (LSD) t-test was conducted for comparison of differences at different time points within groups. $\mathrm{P}<0.05$ indicates that the difference is statistically significant. 
Table I. Demographics and general clinical data of all studied patients.

\begin{tabular}{|c|c|c|c|}
\hline Parameters & $\begin{array}{l}\text { Combination group } \\
n=65\end{array}$ & $\begin{array}{l}\text { Ranibizumab group } \\
n=65\end{array}$ & P-value \\
\hline Sex (male/female) & $37 / 28$ & $33 / 32$ & 0.598 \\
\hline Age (years) & $58.53 \pm 10.38$ & $59.74 \pm 10.76$ & 0.515 \\
\hline Course of the disease (months) & $11.8 \pm 7.3$ & $13.5 \pm 8.1$ & 0.211 \\
\hline BCVA (logMAR) & $0.88 \pm 0.44$ & $0.91 \pm 0.39$ & 0.412 \\
\hline $\mathrm{CMT}(\mu \mathrm{m})$ & $403.6 \pm 56.7$ & $393.1 \pm 74.8$ & 0.369 \\
\hline IOP (mmHg) & $14.6 \pm 3.7$ & $15.0 \pm 3.4$ & 0.522 \\
\hline Type & & & 0.602 \\
\hline Predominantly classic CNV & $31(47.7 \%)$ & $28(43.1 \%)$ & \\
\hline Minimally classic CNV & $20(30.8 \%)$ & $18(27.7 \%)$ & \\
\hline Occult CNV & $14(21.5 \%)$ & $19(29.2 \%)$ & \\
\hline Location & & & 0.474 \\
\hline Subfoveal & $38(58.5 \%)$ & $43(66.2 \%)$ & \\
\hline Juxtafoveal & $19(29.2 \%)$ & $13(20.0 \%)$ & \\
\hline Extrafoveal & $8(12.3 \%)$ & $9(13.8 \%)$ & \\
\hline
\end{tabular}

$\log$ MAR, logarithm of the minimal angle resolution; BCVA, best corrected visual acuity; CMT, central macular thickness; IOP, intraocular pressure; $\mathrm{CNV}$, choroidal neovascularization.

\section{Results}

Changes in BCVA before and after treatment in the two groups of patients. There were statistically significant changes in the BCVA before and after treatment in the two groups of patients $\left(\mathrm{F}_{\text {group }}=17.96, \mathrm{~F}_{\text {time }}=11.89, \mathrm{~F}_{\text {time }} \times\right.$ group $\left._{\mathrm{e}}=16.34, \mathrm{P}<0.01\right)$. The BCVA at 1, 3, 6 and 12 months after treatment was superior to that before treatment in both groups $(\mathrm{P}<0.05)$. As for comparisons between the two groups at 1, 3, 6 and 12 months after treatment, combination therapy group had remarkably better BCVA than ranibizumab group $(\mathrm{P}=0.041, \mathrm{P}=0.007, \mathrm{P}<0.001$, $\mathrm{P}=0.004)$ (Table II).

Changes in CMT before and after treatment in the two groups of patients. The differences in CMT were statistically significant before and after treatment in the two groups of patients $\left(\mathrm{F}_{\text {groupe }}=10.81, \mathrm{~F}_{\text {timee }}=19.29, \mathrm{~F}_{\text {time }} \times\right.$ group $\left._{\mathrm{e}}=22.79, \mathrm{P}<0.01\right)$ The CMT was decreased markedly at $1,3,6$ and 12 months after treatment in both groups compared with that before treatment $(\mathrm{P}<0.05)$. Combination therapy group exhibited remarkably smaller CMT than ranibizumab group at 1, 3, 6 and 12 months after treatment $(\mathrm{P}=0.016, \mathrm{P}<0.001, \mathrm{P}=0.005$, $\mathrm{P}<0.001$ ) (Table II and Fig. 1).

Changes in intraocular pressure before and after treatment in the two groups of patients. After treatment, 1 case of intraocular pressure was $>21 \mathrm{mmHg}$ in the combination therapy group. In the ranibizumab group, there were 2 cases with intraocular pressure $>21 \mathrm{mmHg}$. None of the three patients were treated with ocular hypotensive drugs, but they returned to normal after 3 days. The average intraocular pressure in the two groups was $14.9 \pm 4.3$ and $15.2 \pm 4.6 \mathrm{mmHg}$, $14.7 \pm 4.1$ and $14.8 \pm 3.9 \mathrm{mmHg}, 14.6 \pm 4.2$ and $14.9 \pm 4.5 \mathrm{mmHg}$, and $14.5 \pm 4.4$ and $14.7 \pm 3.8 \mathrm{mmHg}$ at $1,3,6$ and 12 months
Table II. Comparison of pretreatment and posttreatment BCVA $(\log$ MAR) and CMT $(\mu \mathrm{m})$ of the studied patients in two different groups.

\begin{tabular}{lcc}
\hline & $\begin{array}{c}\text { Combination } \\
\text { group } \\
\mathrm{n}=65\end{array}$ & $\begin{array}{c}\text { Ranibizumab } \\
\text { group } \\
\mathrm{n}=65\end{array}$ \\
\hline BCVA (logMAR) & & \\
Before treatment & $0.88 \pm 0.44$ & $0.91 \pm 0.39$ \\
1 month after treatment & $0.80 \pm 0.15$ & $0.86 \pm 0.18$ \\
3 months after treatment & $0.71 \pm 0.17$ & $0.80 \pm 0.20$ \\
6 months after treatment & $0.61 \pm 0.14$ & $0.72 \pm 0.19$ \\
12 months after treatment & $0.57 \pm 0.22$ & $0.67 \pm 0.17$ \\
CMT ( $\mu$ m) & & \\
Before treatment & $403.6 \pm 56.7$ & $393.1 \pm 74.8$ \\
1 month after treatment & $343.2 \pm 41.4$ & $360.6 \pm 40.1$ \\
3 months after treatment & $305.8 \pm 35.8$ & $329.9 \pm 36.5$ \\
6 months after treatment & $281.1 \pm 31.9$ & $297.4 \pm 33.6$ \\
12 months after treatment & $260.2 \pm 29.3$ & $278.2 \pm 28.8$
\end{tabular}

$\log$ MAR, logarithm of the minimal angle resolution; BCVA, best corrected visual acuity; CMT, central macular thickness.

after treatment, respectively. No statistically significant difference in the intraocular pressure was observed between the two groups before and after treatment $(\mathrm{P}>0.05)$.

Comparison of the area of macular degeneration and CNV leakage before and after treatment between the two groups of patients. FFA showed that the area of macular degeneration 

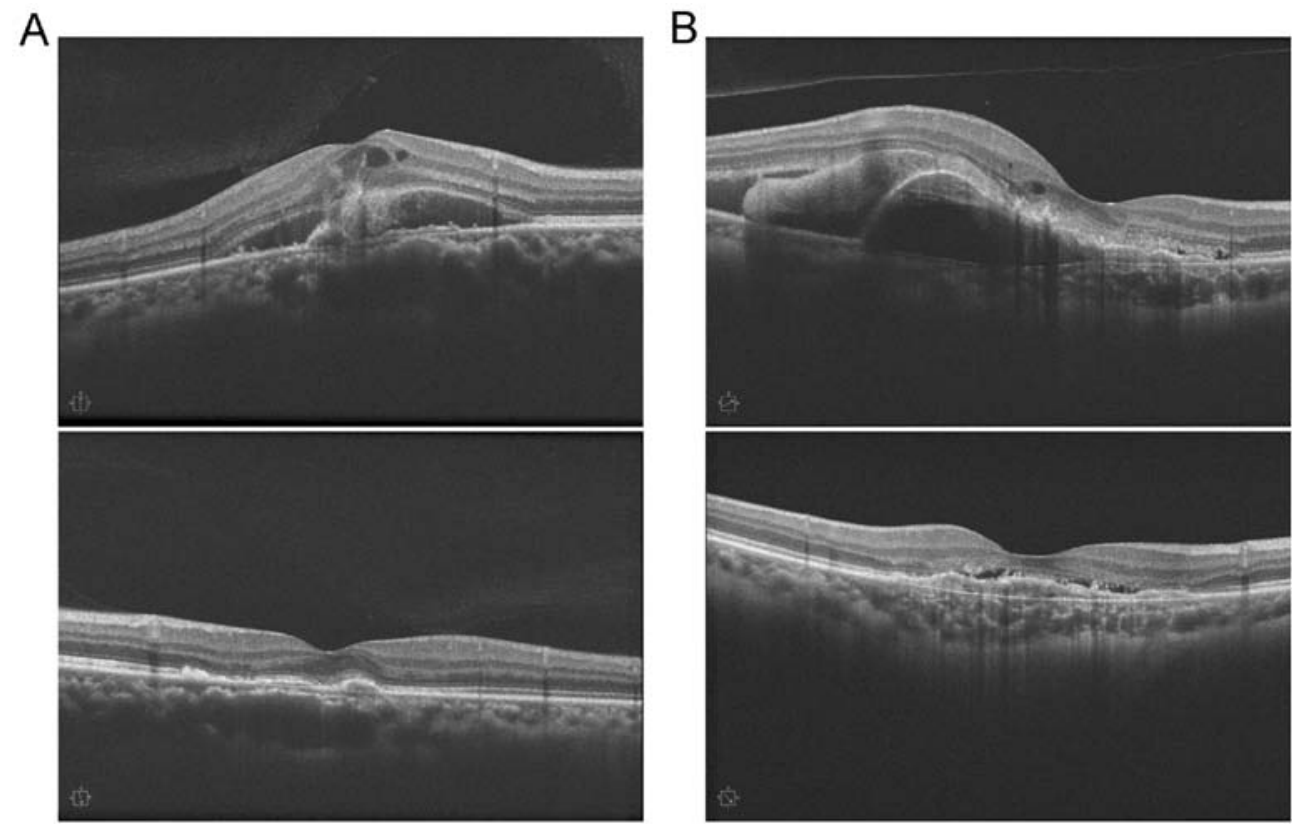

Figure 1. Comparison of pretreatment and 6 months posttreatment OCT images of exudative age-related macular degeneration patients in combination group (A) and ranibizumab group (B). Pretreatment OCT images indicate severe neuroepithelium detachment and macular edema. OCT images 6 months after treatment show CMT dramatically decreased versus pretreatment in both groups. OCT, optical coherence tomography; CMT, central macular thickness.
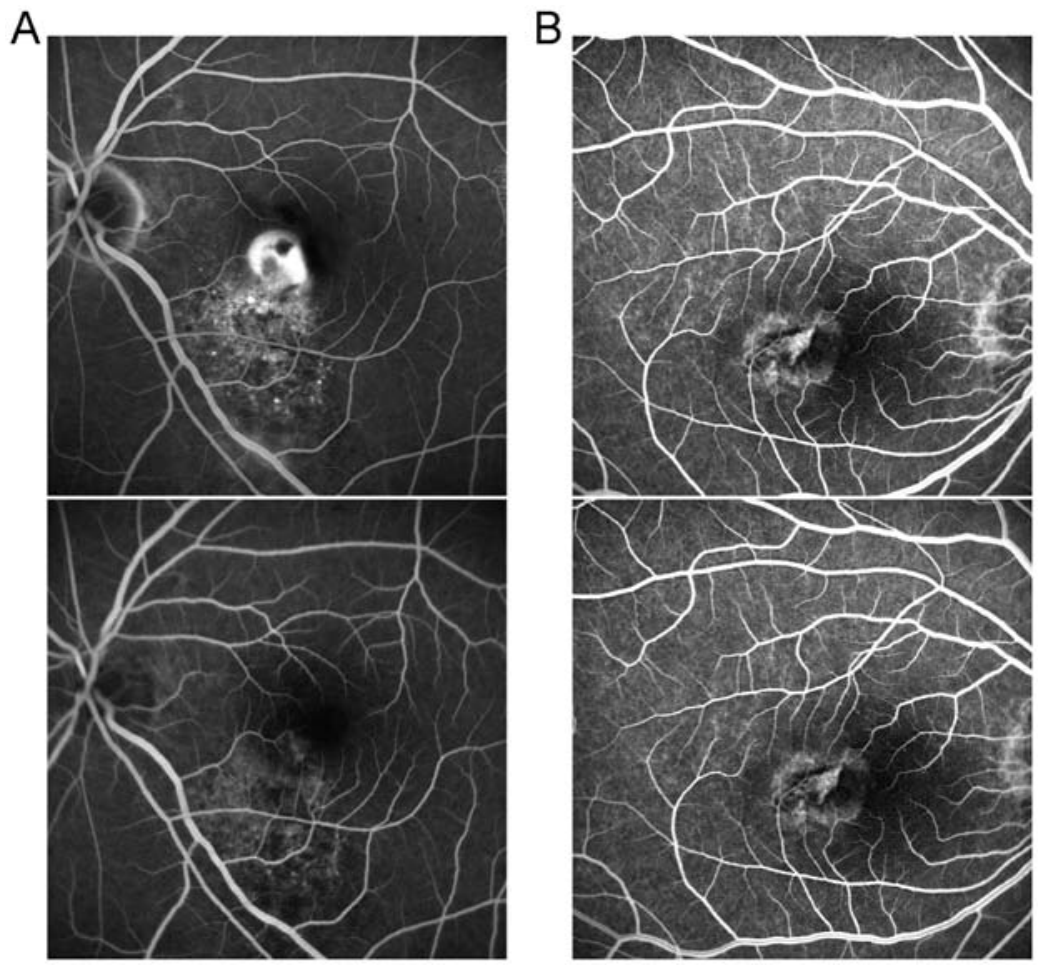

Figure 2. Comparison of pretreatment and 6-month posttreatment FFA images of exudative age-related macular degeneration patients in combination group (A) and ranibizumab group (B). The maculopathy area 6 months after treatment were significantly decreased compared to pretreatment in both groups. FFA, fundus fluorescein angiography.

before treatment was $3.6 \pm 0.6$ and $3.5 \pm 0.7 \mathrm{~mm}^{2}(\mathrm{P}=0.384)$. After treatment, the area of macular degeneration was reduced notably in both groups, and repeated measures analysis of variance indicated that there were statistically significant differences in the area of macular degeneration at different time points $\left(\mathrm{F}_{\text {time }}=8.21, \mathrm{P}<0.001\right)$ and between the two groups
$\left(\mathrm{F}_{\text {group }}=12.42, \mathrm{P}<0.001\right)$. Moreover, the area of macular degeneration in combination therapy group was evidently smaller than that in ranibizumab group at 1,3 and 6 months after treatment $(\mathrm{P}=0.047, \mathrm{P}=0.025, \mathrm{P}=0.027)$, while it manifested no statistically significant difference between the two groups at 12 months after treatment ( $\mathrm{P}=0.083)$ (Table III and Fig. 2). 
A



B

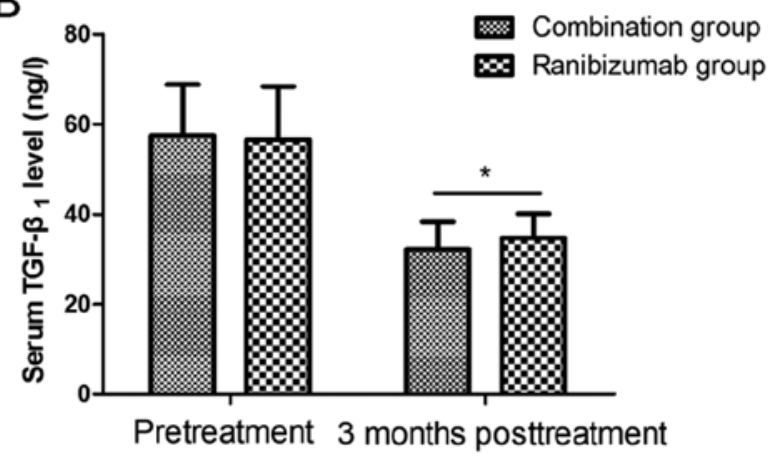

Figure 3. The serum level of VEGF (A) and TGF- $\beta 1$ (B) 3 months after treatment were significantly decreased compared to pretreatment in patients of both groups $(\mathrm{P}<0.05)$. The serum VEGF (A) and TGF- $\beta 1$ (B) level 3 months after treatment of patients in combination group were significantly lower than those of patients in ranibizumab group $(\mathrm{P}=0.024, \mathrm{P}=0.015){ }^{*} \mathrm{P}<0.05$. VEGF, vascular endothelial growth factor; TGF- $\beta 1$, transforming growth factor- $\beta 1$.

Table III. Comparison of pretreatment and posttreatment maculopathy area $\left(\mathrm{mm}^{2}\right)$ of the studied patients in two groups.

\begin{tabular}{lcc}
\hline & $\begin{array}{c}\text { Combination } \\
\text { group } \\
\mathrm{n}=65\end{array}$ & $\begin{array}{c}\text { Ranibizumab } \\
\text { group } \\
\mathrm{n}=65\end{array}$ \\
\hline Maculopathy area $\left(\mathrm{mm}^{2}\right)$ & & \\
Before treatment & $3.6 \pm 0.6$ & $3.5 \pm 0.7$ \\
1 month after treatment & $2.3 \pm 0.8$ & $2.6 \pm 0.9$ \\
3 months after treatment & $2.2 \pm 0.7$ & $2.5 \pm 0.8$ \\
6 months after treatment & $2.0 \pm 0.6$ & $2.3 \pm 0.9$ \\
12 months after treatment & $2.1 \pm 0.6$ & $2.3 \pm 0.7$ \\
\hline
\end{tabular}

According to the statistics of FFA results, there were 31 cases of stable, 14 cases of improved, 16 cases of ameliorated and 4 cases of progressive CNV leakage at 12 months after treatment in combination therapy group, while the numbers of cases were $25,18,11$ and 11 , respectively, in ranibizumab group. The effective rate was $93.8 \%$ in combination therapy group and $83.1 \%$ in ranibizumab group. The difference in the CNV leakage at 12 months after treatment was not statistically significant between the two groups $(\mathrm{P}=0.097)$.

Incidence of treatment-related complications in the two groups of patients. Among the 65 patients receiving PDT, 2 had transient back pain that disappeared within several minutes after the termination of drug injection. A minority of patients had temporary blurred vision, which was improved 1 week later, without severe hypopsia. There was 1 patient in ranibizumab group who had a small amount of macular hemorrhage, which was absorbed in a short term.

Comparison of serum VEGF and TGF- $\beta 1$ concentrations between the two groups of patients. The levels of serum VEGF and TGF- $\beta 1$ displayed no statistically significant differences before treatment between the two groups $(\mathrm{P}=0.241, \mathrm{P}=0.658)$. At 3 months after treatment, the level of serum VEGF declined from $161.3 \pm 11.6$ to $75.9 \pm 9.3 \mathrm{ng} / 1$ and from $158.8 \pm 12.6$ to $79.6 \pm 9.1 \mathrm{ng} / 1$, and that of TGF- $\beta 1$ was reduced from
$57.5 \pm 11.3$ to $32.2 \pm 6.1 \mathrm{ng} / 1$ and from $56.6 \pm 11.8$ to $34.7 \pm 5.4 \mathrm{ng} / 1$ in the two groups. The levels were distinctly lower after treatment than those before treatment $(\mathrm{P}<0.05)$, and they were prominently decreased in combination therapy group in comparison with those in ranibizumab group $(\mathrm{P}=0.024$, $\mathrm{P}=0.015)$ (Fig. 3)

\section{Discussion}

Currently, the commonly applied therapeutic methods for exudative AMD include traditional laser photocoagulation, PDT, TTT, anti-VEGF drug therapy, glucocorticoid drug therapy, surgical treatment, radiotherapy and gene therapy, but none of them can completely cure CNV, and repeated treatments are necessary, thereby increasing the treatment costs and complications (9). The disturbed balance between pro-angiogenic and anti-angiogenic factors is the main cause of CNV, and anti-VEGF drugs have become the preferred therapy (10). As a kind of high-affinity recombinant humanized monoclonal antibody-binding fragment (Fab), ranibizumab has relatively strong affinity to all VEGF-A subunits, and it can inhibit neovascularization, block secondary vascular effects, effectively prevent vascular leakage and control disease progression through competitively binding to VEGF (11).

PDT, a selective treatment of CNV, is the major clinical treatment method for wAMD at present, which selectively acts on neovascularization by virtue of photosensitizer and produces photochemical reactions via laser irradiation to shrink the neovascularization, thus achieving the therapeutic purpose. PDT possesses clinical features such as good tissue selectivity, superficial action, strong damage effects on microvascular tissues, topical treatment and few systemic side effects $(12,13)$. The study of Spielberg et al (14) indicated that PDT combined with IVR is safe and efficacious in treating macular CNV. Nakamura et al (15) treated 38 eyes of 38 AMD patients with ranibizumab combined with PDT (intravitreal injection of $0.5 \mathrm{mg}$ of ranibizumab, execution of PDT of standard dose within 1 week and the second intravitreal injection 1 month later). The follow-up results manifested that the majority of patients (94.8\%) had remarkably ameliorated visual acuity and central foveal thickness as well as a low recurrence rate. Saviano et al (16) proved that PDT combined with intravitreal injection of 
bevacizumab is superior to monotherapy in treating macular CNV. A randomized, two-way clinical trial of 36 patients with exudative AMD was performed by Potter et al (17). The first group received bevacizumab in combination with V-PDT $\left(25 \mathrm{~J} / \mathrm{cm}^{2}\right)$, the second group received bevacizumab in combination with reduced PDT $\left(12 \mathrm{~J} / \mathrm{cm}^{2}\right)$, and the third group received only bevacizumab. Based on the OCT results of the monthly follow-up after the initial treatment, it was decided whether to re-inject bevacizumab or re-administer the combination for 6 months. The test results showed that the patients need 2.8, 2.5 and 5.1 times of bevacizumab injection on average in group I, group II and group III, respectively, so it can be seen that the frequency of injection in group I and group II is notably lower than that in group III $(\mathrm{P}<0.01)$ (17). Kim et al (18) also confirmed that V-PDT combined with 3 successive times of bevacizumab injection can distinctly improve the visual acuity and reduce CMT.

In this study, it was revealed that ranibizumab combined with PDT was able to prominently enhance the BCVA and decrease CMT of the AMD patients in comparison with ranibizumab monotherapy. The area of macular degeneration was reduced after treatment, and it was evidently smaller in combination therapy group than that in ranibizumab group, suggesting that the combination therapy can strengthen the improvement effect on macular morphology more favorably. In terms of improving CNV leakage, combination therapy group was more effective than ranibizumab group, but the difference was not statistically significant, which may be associated with the smaller sample included in this study.

As the most potent pro-angiogenic factor recognized internationally so far, VEGF can enhance the microvascular permeability, increase the release of tissue factors and proteases and stimulate division of endothelial cells. TGF- $\beta 1$ directly regulates VEGF expression and participates in CNV (19-21). According to the results in this study, obviously reduced concentrations of serum VEGF and TGF- $\beta 1$ were observed after combination therapy, implying that the disease is controlled efficiently, and the visual acuity is improved. PDT can block the existing $\mathrm{CNV}$, and ranibizumab is capable of resisting VEGF and repressing CNV, so the clinical efficacy is strengthened apparently by combining the two methods.

In conclusion, the IVR combined with PDT can effectively improve the visual acuity, decrease CMT and prominently reduce the area of macular degeneration of wAMD patients, and its therapeutic effects are long-standing and tolerable for the patients, so it is worthy of clinical popularization.

\section{Acknowledgements}

Not applicable.

\section{Funding}

No funding was received.

\section{Availability of data and materials}

All data generated or analyzed during this study are included in this published article.

\section{Authors' contributions}

LC, BW and SF designed the study and performed the experiments, LC and WC collected the data, BW and WC analyzed the data, LC, BW and SF prepared the manuscript. All authors read and approved the final manuscript.

\section{Ethics approval and consent to participate}

This study was approved by the Ethics Committee of Affiliated to Qingdao University Yuhuangding Hospital of Yantai (Yantai, China). Signed informed consents were obtained from the patients and/or guardians.

\section{Patient consent for publication}

Not applicable.

\section{Competing interests}

The authors declare they have no competing interests.

\section{References}

1. Mehta S: Age-related macular degeneration. Prim Care 42: 377-391, 2015.

2. Wong CW, Yanagi Y, Lee WK, Ogura Y, Yeo I, Wong TY and Cheung CMG: Age-related macular degeneration and polypoidal choroidal vasculopathy in Asians. Prog Retin Eye Res 53: 107-139, 2016.

3. Waldstein SM, Simader C, Staurenghi G, Chong NV, Mitchell P, Jaffe GJ, Lu C, Katz TA and Schmidt-Erfurth U: Morphology and visual acuity in aflibercept and ranibizumab therapy for neovascular age-related macular degeneration in the VIEW trials. Ophthalmology 123: 1521-1529, 2016.

4. Cruess AF, Zlateva G, Pleil AM and Wirostko B: Photodynamic therapy with verteporfin in age-related macular degeneration: A systematic review of efficacy, safety, treatment modifications and pharmacoeconomic properties. Acta Ophthalmol 87: 118-132, 2009.

5. Teper SJ, Nowinska A, Pilat J and Wylegala E: Photodynamic therapy in VEGF inhibition non-responders - Pharmacogenetic study in age-related macular degeneration assessed with swept-source optical coherence tomography. Photodiagn Photodyn Ther 13: 108-113, 2016.

6. Saviano S, Leon PE, Mangogna A and Tognetto D: Combined therapy (intravitreal bevacizumab plus verteporfin photodynamic therapy) versus intravitreal bevacizumab monotherapy for choroidal neovascularization due to age-related macular degeneration: A 1-year follow-up study. Digit J Ophthalmol 22: 46-53, 2016.

7. Su Y, Wu J and Gu Y: Photodynamic therapy in combination with ranibizumab versus ranibizumab monotherapy for wet age-related macular degeneration: A systematic review and meta-analysis. Photodiagn Photodyn Ther 22: 263-273, 2018.

8. Datseris I, Kontadakis GA, Diamanti R, Datseris I, Pallikaris IG, Theodossiadis P and Tsilimbaris MK: Prospective comparison of low-fluence photodynamic therapy combined with intravitreal bevacizumab versus bevacizumab monotherapy for choroidal neovascularization in age-related macular degeneration. Semin Ophthalmol 30: 112-117, 2015.

9. Al-Zamil WM and Yassin SA: Recent developments in age-related macular degeneration: A review. Clin Interv Aging 12: 1313-1330, 2017.

10. Mitchell P, Liew G, Gopinath B and Wong TY: Age-related macular degeneration. Lancet 392: 1147-1159, 2018.

11. Rufai SR, Almuhtaseb H, Paul RM, Stuart BL, Kendrick T, Lee $\mathrm{H}$ and Lotery AJ: A systematic review to assess the 'treat-and-extend' dosing regimen for neovascular age-related macular degeneration using ranibizumab. Eye (Lond) 31: 1337-1344, 2017. 
12. Singh CN and Saperstein DA: Combination treatment with reduced-fluence photodynamic therapy and intravitreal injection of triamcinolone for subfoveal choroidal neovascularization in macular degeneration. Retina 28: 789-793, 2008.

13. Kang EC, Seo JG, Kim BR and Koh HJ: Clinical outcomes of Iintravitreal bevacizumab versus photodynamic therapy with or without bevacizumab for myopic choroidal neovascularization: A 7-year follow-up study. Retina 37: 1775-1783, 2017.

14. Spielberg L and Leys A: Treatment of neovascular age-related macular degeneration with a variable ranibizumab dosing regimen and one-time reduced-fluence photodynamic therapy: The TORPEDO trial at 2 years. Graefes Arch Clin Exp Ophthalmol 248: 943-956, 2010.

15. Nakamura T, Miyakoshi A, Fujita K, Yunoki T, Mitarai K, Yanagisawa S, Fuchizawa C and Hayashi A: One-year results of photodynamic therapy combined with intravitreal ranibizumab for exudative age-related macular degeneration. J Ophthalmol 2012: 154659, 2012.

16. Saviano S, Piermarocchi R, Leon PE, Mangogna A, Zanei A, Cavarzeran Sc F and Tognetto D: Combined therapy with bevacizumab and photodynamic therapy for myopic choroidal neovascularization: A one-year follow-up controlled study. Int J Ophthalmol 7: 335-339, 2014
17. Potter MJ, Claudio CC and Szabo SM: A randomised trial of bevacizumab and reduced light dose photodynamic therapy in age-related macular degeneration: The VIA study. Br J Ophthalmol 94: 174-179, 2010.

18. Kim HW, Kim JL, Lee MH, Yoo HG, Chung IY and Lee JE: Combined treatment of photodynamic therapy and bevacizumab for choroidal neovascularization secondary to age-related macular degeneration. Korean J Ophthalmol 25: 231-237, 2011.

19. Cheung GCM, Lai TYY, Gomi F, Ruamviboonsuk P, Koh A and Lee WK: Anti-VEGF therapy for neovascular AMD and polypoidal choroidal vasculopathy. Asia Pac J Ophthalmol (Phila) 6: 527-534, 2017.

20. Tosi GM, Caldi E, Neri G, Nuti E, Marigliani D, Baiocchi S, Traversi C, Cevenini G, Tarantello A, Fusco F, et al: HTRA1 and TGF- $\beta 1$ concentrations in the aqueous humor of patients with neovascular age-related macular degeneration. Invest Ophthalmol Vis Sci 58: 162-167, 2017.

21. Cabral T, Mello LGM, Lima LH, Polido J, Regatieri CV, Belfort R Jr and Mahajan VB: Retinal and choroidal angiogenesis: A review of new targets. Int J Retina Vitreous 3: 31, 2017

This work is licensed under a Creative Commons Attribution-NonCommercial-NoDerivatives 4.0 International (CC BY-NC-ND 4.0) License. 